Kshitij Chatterjee', Abhinav Goyal', Deepak Reddy', Vikas Koppurapu', Ayoub Innabi', Bashar Alzghoul', Rajani Jagana ${ }^{1}$

'University of Arkansas for Medical Sciences, USA

${ }^{2}$ Einstein Medical Center, Philadelphia, USA

\title{
Prevalence and predictors of readmissions among adults with cystic fibrosis in the United States
}

The authors declare no financial disclosure

\begin{abstract}
Introduction: Patients with Cystic Fibrosis (CF) have increasing rates of hospitalization. We analyzed the burden and predictors of thirty-day readmission among patients with CF in the U.S.

Material and methods: Nationwide Readmission Database (NRD) 2013 was used to identify adults with CF who were hospitalized. These individuals were followed to determine the prevalence of readmission within thirty days of index discharge. Cox proportional hazard regression was used to identify independent predictors of readmission.

Results: There were 14,616 index admissions of adults with CF in 2013. Of these, 2,606 (17.8\%) patients were readmitted within 30 days of discharge. Female sex and chronic anemia were independent predictors of readmission. The most common causes of readmission were pulmonary exacerbation (31\%), lung transplant complications $(5.2 \%)$, and septicemia (3.4\%).

Conclusion: Readmissions are frequent among adults with CF and contribute to significant healthcare burden and cost among this population.
\end{abstract}

Key words: cystic fibrosis, readmissions, epidemiology

Adv Respir Med. 2018; 86: 75-77

\section{Introduction}

Patients with cystic fibrosis (CF) have high rates of hospitalization. In the United States (U.S.), the annual rate of hospitalizations per 1,000 CF patients has increased from 994 to 1072 between 2003 and 2013 [1]. Individuals with CF are prone to frequent readmissions due to pulmonary exacerbation, non-pulmonary comorbidities in $\mathrm{CF}$, or complications related to therapy. These readmissions can impact quality of life of CF patients as well as lead to frequent health exposure and associated complications. There is lack of data on the burden of readmissions among this population. We aim to determine the prevalence of thirty-day readmissions among adults with $\mathrm{CF}$ in the U.S and its predictors.

\section{Material and methods}

We used Nationwide Readmission database (NRD) 2013 which is an all-payer nationally representative database developed by Agency for Healthcare Research and Quality (AHRQ) for Healthcare Cost and Utilization Project (HCUP) [2]. We identified our index admission as the first hospitalization of adult (age $\geq 18$ years) with CF (ICD-9-CM codes 277.00 to 277.09). We identified index admissions and thirty day readmissions based on the criteria of Centers for Medicare and Medicaid Services (CMS) [3]. Any hospitalization within 30 days of an index discharge was considered a readmission, and any admission beyond 30 days of index discharge was a separate index admission. Patients with age $<18$ years and those

Address for correspondence: Kshitij Chatterjee, University of Arkansas for Medical Sciences, USA, kchatterjee@uams.edu 
missing information on key demographics (age and gender) were excluded. We also excluded patients who died during index admission as they were not at risk of readmission and patients with index discharges in December due to lack of sufficient time to capture their thirty-day readmissions [4]. Sampling weights provided were used to produce national level estimates. The primary objective was to determine the prevalence of thirty-day readmissions among hospitalized adults with CF. Secondary objectives were to determine the predictors of readmissions and the common indications for readmissions. We also performed cost and length of stay comparison between index admissions and readmissions. To identify the independent predictors of readmission, Cox proportional hazards regression model was used. The time to the first readmission from index discharge was defined as the time to event. Index hospitalizations without subsequent readmissions within thirty days of discharge were right censored. The covariates included in the model were patient demographics, Elixhauser comorbidities, in-hospital complications, lung transplant status, primary payer, and economic status of the patient (surrogated by median household income quartile of patient's ZIP code).

\section{Results}

Between January and November 2013, there were 14,616 index hospitalizations of adults with CF. Their mean age was 30.4 years (SD 11.6) and $53 \%$ were females. The demographics and co-morbidities are shown in detail in Table 1. Based on CF registry annual report, there were approximately 13,967 adults with CF in the U.S. in 2013 [5]. Our analysis estimates that of these, 8,228 (59\%) individuals were at least hospitalized once during 2013.
Among the 14,616 index admissions, 2,606 $(17.8 \%)$ patients were readmitted within 30 days of index discharge. Compared to patients without readmissions, those who got readmitted had higher proportion of females, had higher prevalence of chronic liver disease $(8.3 \%$ vs $5.6 \%$, p < 0.001), chronic anemia, diabetes, lung transplant recipients, and acute kidney injury (AKI) during index admission (Table 1). However, after multivariate adjustment (listed in methods) on cox proportional hazard regression, the independent predictors for any cause thirty-day readmissions were female sex (Hazard Ratio, HR $=1.19$, $p$ $=0.041)$ and chronic anemia $(\mathrm{HR}=1.52, \mathrm{p}<$ 0.001) (Table 2). Age, lung transplant status, AKI, diabetes, or liver disease did not have any effect on readmission risk.

The median time to the first readmission after index discharge was 15 days. Readmissions had a higher mean cost than index admissions $(\$ 26,274$ vs $\$ 20062, p<0.001)$ despite similar length of stay (median $=7$ days in both). The most common reasons for readmissions were pulmonary exacerbation (31\%), lung transplant complications (5.2\%), and septicemia (3.4\%).

\section{Discussion}

This is the first study to report the burden and predictors of readmissions among adults with CF in a nationally representative sample. Our data show that about $18 \%$ of adults with CF who are hospitalized get readmitted within thirty days. This high readmission rate is similar to that of other respiratory conditions with a high burden of readmissions like COPD and pneumonia [4, 6]. The annual number of hospitalizations of patients with $\mathrm{CF}$ as well hospitalizations per thousand CF patients has increased over the past decade

Table 1. Demographic characteristics and comorbidities of index admissions (with and without readmissions). All cells in the table represent percentage distribution unless otherwise specified in first column. P-value in the last column represents the significance of difference of characteristics between index admission without readmission group and index admission with at least 1 readmission group

\begin{tabular}{lcc}
\hline Covariate & Hazard ratio (95\% confidence interval) & p-value \\
\hline Age & $0.99(0.98-1.00)$ & 0.087 \\
Female (male-referent) & $1.19(1.01-1.41)$ & 0.041 \\
Lung transplant & $1.16(0.82-1.65)$ & 0.402 \\
Acute kidney injury & $1.21(0.96-1.54)$ & 0.115 \\
Chronic anemia & $1.52(1.25-1.85)$ & $<0.001$ \\
Diabetes & $1.22(0.99-1.48)$ & 0.054 \\
\hline
\end{tabular}


Table 2. Results of the Cox proportional hazard regression demonstrating the hazard ratio (HR) and $95 \%$ confidence intervals (CI). The HR represents the risk of thirty-day readmission

\begin{tabular}{|c|c|c|c|c|}
\hline & $\begin{array}{l}\text { All index admissions } \\
\quad(n=14,616)\end{array}$ & $\begin{array}{l}\text { Index admission without } \\
\text { readmission } \\
(\mathrm{n}=12,010)\end{array}$ & $\begin{array}{l}\text { Index admission with } \\
\text { readmission } \\
(\mathrm{n}=2,606)\end{array}$ & p-value \\
\hline Mean age, years (SD) & $30.4(11.6)$ & $30.5(11.6)$ & $30.1(11.3)$ & 0.152 \\
\hline \multicolumn{5}{|l|}{ Gender } \\
\hline Male & 46.6 & 47.6 & 42.1 & $<0.001$ \\
\hline Female & 53.4 & 52.4 & 57.9 & \\
\hline Lung transplant (previous or current) & 5.5 & 5.2 & 7.2 & $<0.001$ \\
\hline Acute kidney injury & 6.4 & 5.4 & 11.2 & $<0.001$ \\
\hline Chronic anemia & 14.7 & 12.9 & 23.2 & $<0.001$ \\
\hline Diabetes & 40.4 & 38.8 & 47.7 & $<0.001$ \\
\hline Median length of stay (IOR), days & $7(8)$ & $7(8)$ & $8(9)$ & $<0.001$ \\
\hline
\end{tabular}

[1]. With the increasing age of the CF population and longer survival, the readmissions for adults with CF are expected to increase over time. This might lead to even further increase in the annual rate of hospitalizations among this population. Recognizing patients at high risk (such as those with chronic anemia) and targeted interventions is necessary to reduce readmissions among these patients.

The role of routine follow-up shortly after discharge in reduction of readmissions (similar to patients with congestive heart failure) in CF has not been examined [7]. Theoretically, such a follow up would be beneficial to address any acute symptoms, compliance with various therapies, assess nutritional and psychosocial status following a hospitalization among CF patients. Whether this would lead to a decrease in readmissions needs to be studied. Our study also highlights the key impact of readmissions on healthcare costs. As the readmissions have a higher mean cost compared to index hospitalizations, they contribute to a significant healthcare burden and cost among adults with CF.

The results of our study should be interpreted in the light of several limitations. First, as NRD is an administrative database, the selection of study population might be subjected to coding errors and misclassification. Secondly, lab values and data on severity of CF such as results of pulmonary function tests were not available. Therefore, there remains a risk of residual confounding despite adjusting for multiple variables in the regression model.

\section{Conclusion}

In conclusion, in a nationally representative sample, about $18 \%$ of hospitalized adults with CF were readmitted within thirty days. Females and patients with chronic anemia are at higher risk of readmission. Readmissions had a higher cost of healthcare compared to index admissions among CF patients.

\section{Conflict of interest}

The authors declare no conflict of interest.

\section{References:}

1. Chatterjee K, Goyal A, Shah N, et al. Contemporary national trends of cystic fibrosis hospitalizations and co-morbidities in the United States. Adv Respir Med. 2016; 84(6): 316-323, doi: 10.5603/ARM.2016.0041, indexed in Pubmed: 28009031.

2. Nationwide Readmission Database Overview. https://www. hcup-us.ahrq.gov/nrdoverview.jsp (27.02.2017).

3. Centers for Medicare and Medicaid Services. Measure Methodology [Internet]. 2017. https://www.cms.gov/Medicare/Quality-Initiatives-Patient-assessment-Instruments/HospitalQualityInits/Measure-Methodology.html (27.02.2017).

4. Chatterjee K, Goyal A, Joshi M. Thirty-Day Readmissions in Adults Hospitalized for COPD or Bronchiectasis: Findings From the Nationwide Readmission Database 2013. Chest. 2017; 151(4): 943-945, doi: 10.1016/j.chest.2016.12.034, indexed in Pubmed: 28390634.

5. 2013 CFF Annual Data Report. https://www.cff.org/2013 CFF_Annual_Data_Report_to_the_Center_Directors.pdf (27.02.2017)

6. Dreyer RP, Dharmarajan K, Hsieh AF, et al. Diagnoses and timing of 30-day readmissions after hospitalization for heart failure, acute myocardial infarction, or pneumonia. JAMA. 2013; 309(4): 355-363, doi: 10.1001/jama.2012.216476, indexed in Pubmed: 23340637.

7. Hernandez AF, Greiner MA, Fonarow GC, et al. Relationship between early physician follow-up and 30-day readmission among Medicare beneficiaries hospitalized for heart failure. JAMA. 2010; 303(17): 1716-1722, doi: 10.1001/jama.2010.533, indexed in Pubmed: 20442387. 